\title{
Oscillation of forced second-order neutral delay differential equations
}

Ying Jiang ${ }^{1}$, Youliang $\mathrm{Fu}^{1}$, Haixia Wang ${ }^{2}$ and Tongxing $\mathrm{Li}^{3,4^{*}}$

\section{"Correspondence:}

litongx2007@163.com

${ }^{3}$ LinDa Institute of Shandong

Provincial Key Laboratory of

Network Based Intelligent

Computing, Linyi University, Linyi,

Shandong 276005, P.R. China

${ }^{4}$ School of Informatics, Linyi

University, Linyi, Shandong 276005,

P.R. China

Full list of author information is

available at the end of the article

\begin{abstract}
The objective of this paper is to study oscillation of a forced second-order neutral differential equation. By using the generalized Riccati substitution and integral technique, a new sufficient condition is obtained which insures that all solutions to the studied equation are oscillatory. An illustrative example is included.
\end{abstract}

MSC: $34 \mathrm{C} 10 ; 34 \mathrm{~K} 11$

Keywords: oscillation; second-order; forced term; neutral differential equation

\section{Introduction}

In this paper, we are concerned with the oscillation of a forced second-order nonlinear neutral differential equation

$$
\left(r(t)[x(t)+P(t) x(\tau(t))]^{\prime}\right)^{\prime}+\sum_{i=1}^{m} Q_{i}(t) f_{i}(x(t))+\sum_{j=1}^{l} R_{j}(t) g_{j}(x(\tau(t)))=F(t),
$$

where $t \geq t_{0}>0, m \geq 1$, and $l \geq 1$ are integers. We suppose that the following assumptions are satisfied:

$\left(\mathrm{A}_{1}\right) r \in \mathrm{C}^{1}\left(\left[t_{0}, \infty\right),(0, \infty)\right), P, Q_{i}, R_{j} \in \mathrm{C}\left(\left[t_{0}, \infty\right),[0, \infty)\right), f_{i}, g_{j} \in \mathrm{C}(\mathbb{R}, \mathbb{R}), y f_{i}(y)>0$, and $y g_{j}(y)>0$ for $y \neq 0, i=1,2, \ldots, m$, and $j=1,2, \ldots, l$;

$\left(\mathrm{A}_{2}\right) \tau \in \mathrm{C}\left(\left[t_{0}, \infty\right), \mathbb{R}\right), \tau(t) \leq t$, and $\lim _{t \rightarrow \infty} \tau(t)=\infty$;

$\left(\mathrm{A}_{3}\right)$ there exist constants $\alpha_{i}>0$ and $\beta_{j}>0$ such that $f_{i}(y) / y \geq \alpha_{i}$ and $g_{j}(y) / y \geq \beta_{j}$ for $y \neq 0$, $i=1,2, \ldots, m$, and $j=1,2, \ldots, l$;

(A $\left.A_{4}\right)$ for any $T \geq t_{0}$, there exist $T \leq s_{1}<t_{1} \leq s_{2}<t_{2}$ such that

$$
F(t) \begin{cases}\leq 0, & t \in\left[s_{1}, t_{1}\right] \\ \geq 0, & t \in\left[s_{2}, t_{2}\right]\end{cases}
$$

and

$$
\sum_{j=1}^{l} \beta_{j} R_{j}(t) \geq \sum_{i=1}^{m} \alpha_{i} Q_{i}(t) P(t), \quad t \in\left[s_{1}, t_{1}\right] \cup\left[s_{2}, t_{2}\right]
$$

(c) 2015 Jiang et al. This article is distributed under the terms of the Creative Commons Attribution 4.0 International License (http://creativecommons.org/licenses/by/4.0/), which permits unrestricted use, distribution, and reproduction in any medium, provided you give appropriate credit to the original author(s) and the source, provide a link to the Creative Commons license, and indicate if changes were made. 
Throughout the paper, we define

$$
z(t):=x(t)+P(t) x(\tau(t))
$$

By a solution of (1.1) we mean a function $x \in \mathrm{C}\left(\left[T_{x}, \infty\right), \mathbb{R}\right), T_{x} \geq t_{0}$, which has the property $r z^{\prime} \in \mathrm{C}^{1}\left(\left[T_{x}, \infty\right), \mathbb{R}\right)$ and satisfies $(1.1)$ on $\left[T_{x}, \infty\right)$. We consider only those solutions $x$ of (1.1) which satisfy condition $\sup \{|x(t)|: t \geq T\}>0$ for all $T \geq T_{x}$. We assume that (1.1) possesses such solutions. A solution of (1.1) is called oscillatory if it has arbitrarily large zeros on the interval $\left[T_{x}, \infty\right)$; otherwise, it is termed nonoscillatory.

As is well known, the study of qualitative theory of differential equations is of importance both in theory and applications. For instance, the problems of oscillatory behavior of neutral differential equations have a number of practical applications in the study of distributed networks containing lossless transmission lines which arise in high-speed computers where the lossless transmission lines are used to interconnect switching circuits. For some related contributions on oscillation of various classes of neutral differential equations, we refer the reader to [1-23] and the references cited therein.

In what follows, we provide some background details that motivated our study. El-Sayed [4] and Wong [19] investigated the second-order forced linear differential equation

$$
\left(p(t) x^{\prime}\right)^{\prime}+q(t) x=f(t) .
$$

Zhang et al. [22] studied a second-order neutral differential equation

$$
\left(r(t)[x(t)+p(t) x(t-\tau)]^{\prime}\right)^{\prime}+Q_{1}(t) f(x(t))+Q_{2}(t) g(x(t-\tau))=H(t)
$$

where $Q_{1}$ and $Q_{2}$ are nonnegative functions. Equation (1.4) is a special case of (1.1). In the sequel, using a generalized Riccati substitution which differs from those exploited in $[4,19,22]$, a new oscillation criterion for $(1.1)$ is presented. Furthermore, an illustrative example is provided.

\section{Main results}

Theorem 2.1 Assume that conditions $\left(\mathrm{A}_{1}\right)-\left(\mathrm{A}_{4}\right)$ are satisfied and let $B_{k}=\left\{u \in C^{1}\left[s_{k}, t_{k}\right]\right.$ : $\left.u(t) \not \equiv 0, u\left(s_{k}\right)=u\left(t_{k}\right)=0\right\}, k=1$, 2. If there exist functions $u \in B_{k}, \rho \in \mathrm{C}^{1}\left(\left[t_{0}, \infty\right),(0, \infty)\right)$, and $\sigma \in \mathrm{C}^{1}\left(\left[t_{0}, \infty\right), \mathbb{R}\right)$ such that, for $k=1,2$,

$$
\begin{aligned}
& J_{k}(u, \rho, \sigma) \\
& \quad=\int_{s_{k}}^{t_{k}}\left\{\rho\left[u^{2}\left(\sum_{i=1}^{m} \alpha_{i} Q_{i}+r \sigma^{2}-(r \sigma)^{\prime}\right)-r\left(u^{\prime}+\frac{u \rho^{\prime}}{2 \rho}+u \sigma\right)^{2}\right]\right\}(t) \mathrm{d} t>0,
\end{aligned}
$$

then every solution of (1.1) is oscillatory.

Proof Suppose that $x$ is a nonoscillatory solution of (1.1) which is eventually positive. Then $z$ defined by (1.3) is also eventually positive. Using $\left(\mathrm{A}_{4}\right)$, for any $T \geq t_{0}$, there exist $t_{1}>s_{1} \geq$ $T$ such that $F(t) \leq 0$ for $t \in\left[s_{1}, t_{1}\right]$. From $\left(\mathrm{A}_{3}\right),(1.1),(1.2)$, and (1.3), we have 


$$
\begin{aligned}
\left(r z^{\prime}\right)^{\prime}(t) & =F(t)-\sum_{i=1}^{m} Q_{i}(t) f_{i}(x(t))-\sum_{j=1}^{l} R_{j}(t) g_{j}(x(\tau(t))) \\
& \leq-\sum_{i=1}^{m} \alpha_{i} Q_{i}(t) x(t)-\sum_{j=1}^{l} \beta_{j} R_{j}(t) x(\tau(t)) \\
& \leq-\left[\sum_{i=1}^{m} \alpha_{i} Q_{i}(t) x(t)+\sum_{i=1}^{m} \alpha_{i} Q_{i}(t) P(t) x(\tau(t))\right] \\
& =-\sum_{i=1}^{m} \alpha_{i} Q_{i}(t) z(t) .
\end{aligned}
$$

For $t \geq T$, we define a generalized Riccati substitution by

$$
V(t):=-\rho(t)\left[\frac{r(t) z^{\prime}(t)}{z(t)}+r(t) \sigma(t)\right]
$$

Then we have

$$
\begin{aligned}
V^{\prime} & =-\rho^{\prime}\left(\frac{r z^{\prime}}{z}+r \sigma\right)-\rho\left(\frac{r z^{\prime}}{z}+r \sigma\right)^{\prime} \\
& =\frac{\rho^{\prime}}{\rho} V-\rho\left(\frac{r z^{\prime}}{z}\right)^{\prime}-\rho(r \sigma)^{\prime} \\
& =\frac{\rho^{\prime}}{\rho} V-\rho \frac{\left(r z^{\prime}\right)^{\prime}}{z}+\rho \frac{r\left(z^{\prime}\right)^{2}}{z^{2}}-\rho(r \sigma)^{\prime} .
\end{aligned}
$$

By virtue of (2.3), we obtain

$$
\left(\frac{z^{\prime}}{z}\right)^{2}=\left(\frac{V}{-\rho r}-\sigma\right)^{2}=\left(\frac{V}{\rho r}\right)^{2}+\sigma^{2}+2 \frac{V \sigma}{\rho r} .
$$

For $t \in\left[s_{1}, t_{1}\right]$, substituting (2.2) and (2.5) into (2.4), we conclude that

$$
\begin{aligned}
V^{\prime} & =\frac{\rho^{\prime}}{\rho} V-\rho \frac{\left(r z^{\prime}\right)^{\prime}}{z}+\rho r\left(\frac{V^{2}}{\rho^{2} r^{2}}+\sigma^{2}+2 \frac{V \sigma}{\rho r}\right)-\rho(r \sigma)^{\prime} \\
& =-\rho \frac{\left(r z^{\prime}\right)^{\prime}}{z}+\rho\left[r \sigma^{2}-(r \sigma)^{\prime}\right]+\left(\frac{\rho^{\prime}}{\rho}+2 \sigma\right) V+\frac{V^{2}}{\rho r} \\
& \geq \rho\left[\sum_{i=1}^{m} \alpha_{i} Q_{i}+r \sigma^{2}-(r \sigma)^{\prime}\right]+\left(\frac{\rho^{\prime}}{\rho}+2 \sigma\right) V+\frac{V^{2}}{\rho r} .
\end{aligned}
$$

Let $u \in B_{1}$ be given as in the hypothesis. Multiplying (2.6) by $u^{2}$ and integrating the resulting inequality from $s_{1}$ to $t_{1}$, we have

$$
\begin{aligned}
\int_{s_{1}}^{t_{1}} u^{2} V^{\prime} \mathrm{d} t \geq & \int_{s_{1}}^{t_{1}} u^{2} \rho\left[\sum_{i=1}^{m} \alpha_{i} Q_{i}+r \sigma^{2}-(r \sigma)^{\prime}\right] \mathrm{d} t+\int_{s_{1}}^{t_{1}}\left(\frac{\rho^{\prime}}{\rho}+2 \sigma\right) V u^{2} \mathrm{~d} t \\
& +\int_{s_{1}}^{t_{1}} \frac{V^{2}}{\rho r} u^{2} \mathrm{~d} t .
\end{aligned}
$$


Integrating (2.7) by parts and using the fact that $u\left(s_{1}\right)=u\left(t_{1}\right)=0$, we deduce that

$$
\begin{aligned}
-\int_{s_{1}}^{t_{1}} 2 u u^{\prime} V \mathrm{~d} t \geq & \int_{s_{1}}^{t_{1}} u^{2} \rho\left[\sum_{i=1}^{m} \alpha_{i} Q_{i}+r \sigma^{2}-(r \sigma)^{\prime}\right] \mathrm{d} t \\
& +\int_{s_{1}}^{t_{1}}\left(\frac{\rho^{\prime}}{\rho}+2 \sigma\right) V u^{2} \mathrm{~d} t+\int_{s_{1}}^{t_{1}} \frac{V^{2}}{\rho r} u^{2} \mathrm{~d} t .
\end{aligned}
$$

That is,

$$
\int_{s_{1}}^{t_{1}}\left[\frac{u^{2} V^{2}}{\rho r}+2 u V\left(u^{\prime}+u\left(\frac{\rho^{\prime}}{2 \rho}+\sigma\right)\right)\right] \mathrm{d} t+\int_{s_{1}}^{t_{1}} u^{2} \rho\left[\sum_{i=1}^{m} \alpha_{i} Q_{i}+r \sigma^{2}-(r \sigma)^{\prime}\right] \mathrm{d} t \leq 0 .
$$

Hence,

$$
\begin{aligned}
& \int_{s_{1}}^{t_{1}}\left[\frac{u V}{\sqrt{\rho r}}+\sqrt{\rho r}\left(u^{\prime}+\frac{u \rho^{\prime}}{2 \rho}+u \sigma\right)\right]^{2} \mathrm{~d} t \\
& \quad+\int_{s_{1}}^{t_{1}}\left[u^{2} \rho\left(\sum_{i=1}^{m} \alpha_{i} Q_{i}+r \sigma^{2}-(r \sigma)^{\prime}\right)-\rho r\left(u^{\prime}+\frac{u \rho^{\prime}}{2 \rho}+u \sigma\right)^{2}\right] \mathrm{d} t \leq 0
\end{aligned}
$$

which is equivalent to

$$
\int_{s_{1}}^{t_{1}}\left[\frac{u V}{\sqrt{\rho r}}+\sqrt{\rho r}\left(u^{\prime}+\frac{u \rho^{\prime}}{2 \rho}+u \sigma\right)\right]^{2} \mathrm{~d} t+J_{1}(u, \rho, \sigma) \leq 0,
$$

where $J_{1}(u, \rho, \sigma)$ is as in (2.1). Since $J_{1}(u, \rho, \sigma)>0$, inequality (2.8) yields

$$
\int_{s_{1}}^{t_{1}}\left[\frac{u V}{\sqrt{\rho r}}+\sqrt{\rho r}\left(u^{\prime}+\frac{u \rho^{\prime}}{2 \rho}+u \sigma\right)\right]^{2} \mathrm{~d} t \leq-J_{1}(u, \rho, \sigma)<0,
$$

which is a contradiction. This contradiction proves that $x$ is not eventually positive.

When $x$ is eventually negative, we use $u \in B_{2}$ and $F(t) \geq 0$ on $\left[s_{2}, t_{2}\right]$ to arrive at a similar contradiction. The proof is complete.

Example 2.1 For $t \geq 1$, consider the forced second-order neutral delay differential equation

$$
\left(x(t)+\frac{1}{2} x\left(\frac{t}{2}\right)\right)^{\prime \prime}+8 x(t)+4 t^{2} x\left(\frac{t}{2}\right)=\sin t .
$$

Let $r(t)=1, P(t)=1 / 2, \tau(t)=t / 2, m=l=1, Q_{1}(t)=8, R_{1}(t)=4 t^{2}, f_{1}(y)=g_{1}(y)=y$, $\alpha_{1}=\beta_{1}=1, u=\sin t, \rho(t)=1$, and $\sigma(t)=0$. Set $s_{1}=(2 n+1) \pi, t_{1}=(2 n+2) \pi, s_{2}=(2 n+3) \pi$, and $t_{2}=(2 n+4) \pi$. Then

$$
\begin{aligned}
J_{1}(u, \rho, \sigma) & =\int_{s_{1}}^{t_{1}}\left\{\rho\left[u^{2}\left(\sum_{i=1}^{m} \alpha_{i} Q_{i}+r \sigma^{2}-(r \sigma)^{\prime}\right)-r\left(u^{\prime}+\frac{u \rho^{\prime}}{2 \rho}+u \sigma\right)^{2}\right]\right\}(t) \mathrm{d} t \\
& =\int_{(2 n+1) \pi}^{(2 n+2) \pi}\left(8 \sin ^{2} t-\cos ^{2} t\right) \mathrm{d} t=\frac{7}{2} \pi .
\end{aligned}
$$

Similarly, $J_{2}(u, \rho, \sigma)=7 \pi / 2$. Hence, by Theorem 2.1 , every solution of (2.9) is oscillatory. 


\section{Competing interests}

The authors declare that they have no competing interests.

\section{Authors' contributions}

All four authors contributed equally to this work. They all read and approved the final version of the manuscript

\section{Author details}

${ }^{1}$ Qingdao Technological University, Feixian, Shandong 273400, P.R. China. ${ }^{2}$ College of Economics, Ocean University of China, Qingdao, Shandong 266100, P.R. China. ${ }^{3}$ LinDa Institute of Shandong Provincial Key Laboratory of Network Based Intelligent Computing, Linyi University, Linyi, Shandong 276005, P.R. China. ${ }^{4}$ School of Informatics, Linyi University, Linyi, Shandong 276005, P.R. China.

\section{Acknowledgements}

The authors are grateful to the editors and two anonymous referees for a very thorough reading of the manuscript and for kindly prompting improvements in presentation. This research is supported by NNSF of P.R. China (Grant No. 61403061), NSF of Shandong Province (Grant No. ZR2012FL06), and the AMEP of Linyi University, P.R. China.

\section{Received: 9 December 2014 Accepted: 26 June 2015 Published online: 18 July 2015}

\section{References}

1. Baculíková, B, Džurina, J: Oscillation theorems for second-order nonlinear neutral differential equations. Comput. Math. Appl. 62, 4472-4478 (2011)

2. Baculíková, B, Džurina, J: Oscillation theorems for higher order neutral differential equations. Appl. Math. Comput. 219, 3769-3778 (2012)

3. Baculíková, B, Džurina, J, Li, T: Oscillation results for even-order quasilinear neutral functional differential equations. Electron. J. Differ. Equ. 2011, 143 (2011)

4. El-Sayed, MA: An oscillation criterion for a forced second order linear differential equation. Proc. Am. Math. Soc. 118, 813-817 (1993)

5. Erbe, L, Kong, Q, Zhang, BG: Oscillation Theory for Functional Differential Equations. Dekker, New York (1995)

6. Grace, SR, Lalli, BS: Oscillation of nonlinear second order neutral delay differential equations. Rad. Mat. 3, 77-84 (1987)

7. Győri, I, Ladas, G: Oscillation Theory of Delay Differential Equations with Applications. Clarendon Press, Oxford (1991)

8. Hale, JK: Theory of Functional Differential Equations. Springer, New York (1977)

9. Hasanbulli, M, Rogovchenko, YuV: Oscillation criteria for second order nonlinear neutral differential equations. Appl. Math. Comput. 215, 4392-4399 (2010)

10. Ladde, GS, Lakshmikantham, V, Zhang, BG: Oscillation Theory of Differential Equations with Deviating Arguments. Dekker, New York (1987)

11. Li, T, Agarwal, RP, Bohner, M: Some oscillation results for second-order neutral differential equations. J. Indian Math. Soc. 79, 97-106 (2012)

12. Li, T, Agarwal, RP, Bohner, M: Some oscillation results for second-order neutral dynamic equations. Hacet. J. Math. Stat. 41, 715-721 (2012)

13. Li, T, Han, Z, Zhao, P, Sun, S: Oscillation of even-order neutral delay differential equations. Adv. Differ. Equ. 2010, 184180 (2010)

14. Li, T, Rogovchenko, YuV: Asymptotic behavior of higher-order quasilinear neutral differential equations. Abstr. Appl. Anal. 2014, 395368 (2014)

15. Li, T, Rogovchenko, YuV, Zhang, C: Oscillation of second-order neutral differential equations. Funkc. Ekvacioj 56, 111-120 (2013)

16. Shi, W, Wang, P: Oscillatory criteria of a class of second-order neutral functional differential equations. Appl. Math. Comput. 146, 211-226 (2003)

17. Sun, S, Li, T, Han, Z, Li, H: Oscillation theorems for second-order quasilinear neutral functional differential equations. Abstr. Appl. Anal. 2012, 819342 (2012)

18. Wang, $P, W u, Y, C a c c e t t a, ~ L:$ Oscillation criteria for boundary value problems of high-order partial functional differential equations. J. Comput. Appl. Math. 206, 567-577 (2007)

19. Wong, JSW: Oscillation criteria for a forced second-order linear differential equation. J. Math. Anal. Appl. 231, 235-240 (1999)

20. Ye, L, Xu, Z: Oscillation criteria for second-order quasilinear neutral delay differential equations. Appl. Math. Comput. 207, 388-396 (2009)

21. Zhang, C, Agarwal, RP, Bohner, M, Li, T: New oscillation results for second-order neutral delay dynamic equations. Adv. Differ. Equ. 2012, 227 (2012)

22. Zhang, Z, Wang, X, Lin, S, Yu, Y: Oscillation and nonoscillation criteria for second order nonlinear neutral delay differential equations. J. Syst. Sci. Math. Sci. 26, 325-334 (2006) (in Chinese)

23. Zhong, J, Ouyang, Z, Zou, S: An oscillation theorem for a class of second-order forced neutral delay differential equations with mixed nonlinearities. Appl. Math. Lett. 24, 1449-1454 (2011) 